\title{
Variabilidad fenotípica del porcentaje de fibras meduladas en el vellón de alpaca Huacaya
}

\author{
Phenotypic variability of percentage of medullated fibers in Huacaya alpaca fleece \\ Rubén Pinares ${ }^{1}$, Gustavo Augusto Gutiérrez ${ }^{1}$ Alan Cruz ${ }^{2}$, Alonso Burgos², \\ Juan Pablo Gutiérrez
}

\section{Resumen}

\begin{abstract}
El objetivo del estudio fue evaluar la variabilidad fenotípica del porcentaje de fibras meduladas de alpacas Huacaya, así como calcular la correlación fenotípica entre el diámetro medio de fibra (DMF) y el porcentaje de medulación total (PM). Se tomaron muestras de fibra de 36 alpacas Huacaya (machos entre 0.4 y 10.4 años) para determinar el DMF y el PM con el microscopio de proyección. El tipo de médula de las fibras fue considerada como no medulada, fragmentada, discontinua, continua y fuertemente medulada. Se observó una alta variabilidad fenotípica del PM entre alpacas y dentro de las muestras. El promedio \pm desviación estándar del PM y DMF fue $32.56 \pm 18.30 \%$ y $17.58 \pm 2.52 \mu$ m para fibras no meduladas; $29.29 \pm 11.67 \%$ y $21.49 \pm 2.39 \mu \mathrm{m}$ para fibras con medulación fragmentada; $14.01 \pm 10.14 \%$ y $24.04 \pm 2.40 \mu \mathrm{m}$ para fibras con medulación discontinua; 23.90 $\pm 13.01 \%$ y $28.04 \pm 3.10 \mu \mathrm{m}$ para fibras con medulación continua; y $0.59 \pm 0.44 \%$ y $50.85 \pm$ $9.86 \mu \mathrm{m}$ para fibras fuertemente meduladas, respectivamente. La correlación fenotípica entre el PM y el DMF fue 0.80, y entre el PM y el porcentaje de medulación continua fue 0.74 . En conclusión, existe una alta variabilidad fenotípica del PM y de los porcentajes de fibras meduladas continuas, discontinuas y fragmentadas en el vellón de alpaca Huacaya. Las fibras finas tienen una baja frecuencia de médulas fragmentadas y discontinuas, mientras que las fibras más gruesas tienen una mayor frecuencia de médulas discontinuas $\mathrm{y}$ continuas.
\end{abstract}

Palabras clave: alpaca Huacaya; fibra medulada; diámetro de fibra; variación fenotípica

\footnotetext{
${ }^{1}$ Programa de Doctorado en Ciencia Animal, Universidad Nacional Agraria La Molina, Lima, Perú

${ }^{2}$ Fundo Pacomarca - Inca Tops S.A, Arequipa, Perú

${ }^{3}$ Departamento de Producción Animal, Universidad Complutense de Madrid, Madrid, España

${ }^{4}$ E-mail: zoovet_p@hotmail.com
}

Fuente financiera: CONCYTEC y Proyecto VLIR - UNALM «Mejorando los sistemas de producción de alpacas en pastizales en la sierra central del Perú»

Recibido: 1 de octubre de 2018

Aceptado para publicación: 4 de abril de 2019 
The objective of the study was to evaluate the phenotypic variability of the percentage of medullated fibres of Huacaya alpacas, as well as to calculate the phenotypic correlation between the mean fiber diameter (MFD) and the percentage of total medullation (PM). Fibre samples were collected from 36 Huacaya alpacas (0.4-10.4 years old males) to analyse the MFD and the PM with the projection microscope. The type of medulla of the fibres was considered as not medullated, fragmented, discontinuous, continuous and strongly medullated. A high phenotypic variability of PM between alpacas and within the samples was observed. The mean \pm standard deviation of PM and MFD was 32.56 $\pm 18.30 \%$ and $17.58 \pm 2.52 \mu \mathrm{m}$ for non-medullated fibres; $29.29 \pm 11.67 \%$ and $21.49 \pm$ $2.39 \mu \mathrm{m}$ for fragmented medullated fibres; $14.01 \pm 10.14 \%$ and $24.04 \pm 2.40 \mu \mathrm{m}$ for discontinuous medullated fibres; $23.90 \pm 13.01 \%$ and $28.04 \pm 3.10 \mu \mathrm{m}$ for continuous medullated fibres; and $0.59 \pm 0.44 \%$ and $50.85 \pm 9.86 \mu \mathrm{m}$ for strongly medullated fibres, respectively. The phenotypic correlation between the PM and the MFD was 0.80, and between the PM and the percentage of continuous medullation was 0.74 . In conclusion, there is a high phenotypic variability of the PM and the percentage of continuous, discontinuous and fragmented medullated fibres in the Huacaya alpaca fleece. The fine fibres have a low frequency of fragmented and discontinuous medullated fibres, while the thicker fibres have a higher frequency of discontinuous and continuous medullated fibres.

Key words: Huacaya alpaca; medullated fibre; fibre diameter; phenotypic variation

\section{INTRODUCCIÓN}

La fibra de alpaca es considerada de alto valor textil debido a su suavidad, largo de mecha y finura; sin embargo, se ha observado en el vellón de la alpaca la presencia de fibras objetables que afectan la calidad de los productos textiles. Las fibras meduladas son consideradas como objetables debido a que afectaría el confort de la prenda y su uniformidad en el teñido. Por otro lado, la presencia de fibras meduladas favorecería la termorregulación de la alpaca en climas fríos, debido a la retención de calor en la médula (Wang et al., 2005; Moore et al., 2011).

Se ha reportado una amplia variabilidad fenotípica del porcentaje de fibras meduladas en los vellones de alpacas (Newman y Paterson, 1996; McGregor, 2006; Lupton et al., 2006), la cual es mayor con respecto a la que se observa en la lana de ovino Corriedale y Romney Marsh (Villarroel, 1963; Sienra et al., 2011; Scobie et al., 2015).
El porcentaje de medulación se incrementa de 10 a $60 \%$ en fibras de 22 y 40 $\mu \mathrm{m}$ (McGregor, 2006), relación que podría ser distinta si se analiza por el tipo de médula de la fibra. Así, la presencia de fibras con médula continua y fibras fuertemente meduladas afectarían la variabilidad del diámetro de fibra en el vellón (Villarroel, 1963; McGregor et al., 2013).

Se han planteado varias alternativas para mejorar la calidad textil de la fibra de alpaca y llama. Una opción es la eliminación de las fibras gruesas mediante el descerdado (Wang et al., 2008; Laime et al., 2016). Sin embargo, la alta variabilidad del porcentaje de fibras meduladas fragmentadas, discontinuas y continuas, aunado a que estas fibras se presentan en un rango amplio de finura (finas a gruesas) en el vellón de la alpaca podría explicar el bajo rendimiento del descerdado (Wang et al., 2008). Por otro lado, la selección es otra alternativa que permitiría reducir el porcentaje de fibras meduladas (Pinares et al., 2018). Así, el presente trabajo tuvo como objetivo determinar la variabilidad fenotípica del porcentaje de fibras 
meduladas en alpacas Huacaya, así como calcular la correlación fenotípica entre el porcentaje de medulación total (PM) con el diámetro medio de fibra total (DMF) y por tipos de médula, con la finura al hilado (FH) y el porcentaje de fibras menores a $30 \mu \mathrm{m}$.

\section{Materiales y Métodos}

Para el cálculo del tamaño muestral se utilizó el método de muestreo probabilístico para una población de 1000 machos, asumiendo un error alfa de 0.05 , una precisión de $9 \%$ para PM y una varianza grande (789) calculada por Cruz et al. (2017) para PM; lo cual resultó en un tamaño muestral de 36 alpacas del ecotipo Huacaya, de sexo macho y con edades entre 0.4 y 10.4 años. Los animales provenían del Centro Genético de Pacomarca, ubicado a $4060 \mathrm{msnm}$, en la provincia de Melgar, departamento de Puno, Perú. Las muestras de fibra fueron tomadas del costillar medio de cada animal, similar a lo reportado por Aylan-Parker y McGregor (2002).

La medición del diámetro individual de fibra y la clasificación del tipo de fibra, según la presencia de medulación, se realizó mediante el método de microscopio de proyección (IWTO-8, 2011) en el Laboratorio de Fibras Textiles, Pieles y Cueros «Alberto Pumayalla Díaz» de la Universidad Nacional Agraria La Molina, Lima. Para cada muestra se registró el diámetro de 600 fibras individuales y se calculó el diámetro medio de fibra total(DMF), la desviación estándar(DE) y el coeficiente de variación del diámetro de fibra $(\mathrm{CV})$ y el porcentaje de fibras menores de $30 \mu \mathrm{m}$. El tipo de fibra fue asignado a los siguientes grupos de acuerdo con la presencia y tipo de médula: no medulada, fragmentada, discontinua, continua y fuertemente medulada (Figura 1).

El número total de fibras meduladas se determinó sumando los grupos de fibra medulada fragmentada, discontinua, continua y fuertemente medulada. El porcentaje de medulación total (PM) fue calculado mediante la fórmula $\mathrm{PM} \%=$ (Número total de fibras

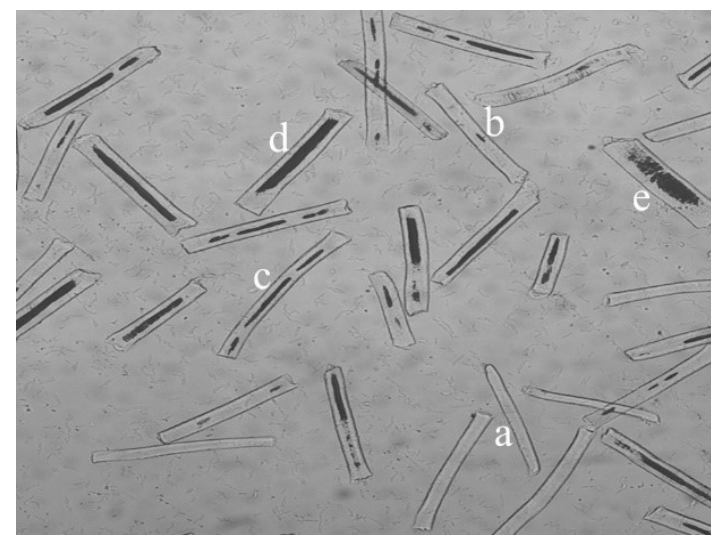

Figura 1. Tipo de fibras en el vellón de alpaca Huacaya: a) no medulada, b) médula fragmentada, c) médula discontinua, d) médula continua, e) fuertemente medulada

meduladas / número total de fibras analizadas) * 100. El error estándar del porcentaje de medulación total (EE) se calculó mediante la fórmula $\mathrm{EE}=\mathrm{v}(\mathrm{m} / \mathrm{n}) *(100-\mathrm{m})$ donde $\mathrm{m}$ es el porcentaje de medulación total y $\mathrm{n}$ es el número total de fibras analizadas.

La finura al hilado de la fibra $(\mathrm{FH})$ se estimó usando la fórmula desarrollada por Butler y Dolling (1995), asumiendo un CV de $24 \%$, donde el valor de la finura al hilado es lo mismo que el DMF. $\mathrm{FH}=0.881 * \mathrm{DMF}^{*}$ $\mathrm{V} 1+5 *(\mathrm{CV} / 100)^{2}$.

El grado de asociación fenotípica entre las características de las fibras meduladas se calculó mediante el coeficiente de correlación de Pearson $(r)$. Además, se estimó los coeficientes de una ecuación de regresión lineal simple para la predicción del PM a partir del DMF. Para el análisis estadístico de los datos se ha usado el paquete estadístico R v. 3.4 .2 (R Core Team, 2017).

\section{Resultados y Discusión}

\section{Finura de la Fibra}

Uno de los principales indicadores de la calidad textil de la fibra de alpaca es el DMF. Este fue de $22.16 \mu \mathrm{m}$, valor ligeramente más 
Cuadro 1. Diámetro medio de fibra (DMF), desviación estándar de DMF (DE), coeficiente de variación de DMF $(\mathrm{CV})$, porcentaje de medulación total $(\mathrm{PM})$, error estándar del PM (EE) y finura al hilado (FH) de fibra de alpacas Huacaya

\begin{tabular}{ccccccc}
\hline $\begin{array}{c}\text { Edad } \\
(\text { años })\end{array}$ & $\begin{array}{c}\text { DMF } \\
(\mu \mathrm{m})\end{array}$ & $\begin{array}{c}\text { DE } \\
(\mu \mathrm{m})\end{array}$ & $\begin{array}{c}\text { CV } \\
(\%)\end{array}$ & $\begin{array}{c}\text { PM } \\
(\%)\end{array}$ & $\begin{array}{c}\text { EE } \\
(\%)\end{array}$ & $\begin{array}{c}\text { FH } \\
(\mu \mathrm{m})\end{array}$ \\
\hline 0.4 & 22.48 & 4.31 & 18.82 & 84.83 & 1.46 & 21.85 \\
0.5 & 21.57 & 5.08 & 23.56 & 62.75 & 1.97 & 21.47 \\
1.4 & 19.98 & 5.15 & 26.09 & 54.17 & 1.75 & 20.37 \\
1.5 & 20.39 & 5.17 & 25.35 & 64.75 & 1.88 & 20.65 \\
1.6 & 21.83 & 5.00 & 22.91 & 63.33 & 1.97 & 21.61 \\
2.4 & 20.88 & 5.26 & 25.22 & 53.71 & 1.91 & 21.12 \\
2.6 & 19.13 & 5.22 & 27.29 & 59.58 & 1.95 & 19.77 \\
3.4 & 25.70 & 4.65 & 18.09 & 83.33 & 1.52 & 24.42 \\
3.6 & 20.45 & 5.60 & 27.60 & 68.75 & 1.77 & 21.13 \\
4.4 & 20.70 & 6.23 & 30.08 & 52.00 & 2.04 & 21.98 \\
5.5 & 24.35 & 5.40 & 22.15 & 74.33 & 1.75 & 23.94 \\
5.7 & 20.74 & 4.89 & 23.58 & 54.83 & 2.03 & 20.66 \\
6.4 & 24.71 & 5.95 & 24.08 & 58.83 & 2.01 & 24.72 \\
6.5 & 26.46 & 6.35 & 24.00 & 87.33 & 1.36 & 26.46 \\
6.8 & 26.07 & 6.78 & 26.02 & 90.83 & 1.18 & 26.57 \\
7.3 & 27.63 & 6.79 & 24.57 & 84.17 & 1.49 & 27.77 \\
7.6 & 22.67 & 5.35 & 23.60 & 85.67 & 1.43 & 22.58 \\
9.2 & 27.56 & 7.27 & 26.38 & 90.50 & 1.20 & 28.19 \\
9.5 & 23.88 & 5.41 & 22.65 & 76.17 & 1.74 & 23.58 \\
10.4 & 26.30 & 6.22 & 23.65 & 91.67 & 1.13 & 26.21 \\
\hline Promedio & 22.16 & 5.42 & 24.64 & 67.44 & 1.74 & 22.28 \\
\hline
\end{tabular}

fino al de $23.07 \mu \mathrm{m}$ reportado por Cervantes et al. (2010) y superior al de $19.9 \mu \mathrm{m}, 20.94$ $\mu \mathrm{m}$ y $21.64 \mu \mathrm{m}$ hallados por Vásquez et al. (2015), Quispe (2010) y González et al. (2008), respectivamente. Estos resultados evidencian la presencia de animales con buena calidad de fibra en el grupo de machos (Cuadro 1). La desviación estándar del DMF fue $5.42 \mu \mathrm{m}$, valor similar a lo reportado por Cervantes et al. (2010) en animales de la misma población.

El valor promedio del CV fue $24.64 \%$, valor similar a lo reportado por Cervantes et al. (2010) y Lupton et al. (2006). Sin embargo, el valor promedio del $\mathrm{CV}$ resultó superior al $18.38 \%, 21.2 \%$ y $21.4 \%$ reportados por González et al. (2008), Vásquez et al. (2015) y Quispe (2010), respectivamente.

El valor promedio de la finura al hilado de la fibra $(\mathrm{FH})$ fue $22.28 \mu \mathrm{m}$, valor superior al de $19.4 \mu \mathrm{m}$ y $20.9 \mu \mathrm{m}$ reportado por Vásquez et al. (2015) y Quispe (2010), respectivamente. McGregor (2006) señala que la finura al hilado está asociado al rendimiento y la uniformidad del hilado, de modo que se prefieren vellones finos y con un valor de $\mathrm{CV}$ menor a $24 \%$. El valor promedio de $\mathrm{FH}$ fue mayor al DMF en $0.12 \mu \mathrm{m}$, diferencia que estaría influenciada por el CV (24.6\%). Los valores altos de $\mathrm{CV}$ en alpacas han sido 
Cuadro 2. Variación fenotípica de fibra medulada continua, discontinua, fragmentada y no medulada $(\%)$ y el diámetro medio de fibra $(\mu \mathrm{m})$ en el vellón de alpaca Huacaya según la edad

\begin{tabular}{cccccccccc}
\hline \multirow{2}{*}{$\begin{array}{c}\text { Edad } \\
\text { (años) }\end{array}$} & $\mathrm{n}$ & \multicolumn{2}{c}{ Continua } & \multicolumn{2}{c}{ Discontinua } & \multicolumn{2}{c}{ Fragmentada } & \multicolumn{2}{c}{ No medulada } \\
\cline { 2 - 10 } & & $\%$ & $\mu \mathrm{m}$ & $\%$ & $\mu \mathrm{m}$ & $\%$ & $\mu \mathrm{m}$ & $\%$ & $\mu \mathrm{m}$ \\
\hline $0.4-0.5$ & 4 & 37.54 & 25.26 & 14.58 & 22.83 & 21.54 & 20.71 & 26.21 & 18.67 \\
$1.4-1.6$ & 8 & 17.58 & 27.43 & 7.04 & 23.20 & 33.21 & 20.87 & 42.04 & 16.64 \\
$2.4-2.6$ & 8 & 19.42 & 26.65 & 10.63 & 22.89 & 26.54 & 20.29 & 43.35 & 16.02 \\
$3.4-4.4$ & 4 & 24.38 & 27.51 & 20.29 & 23.43 & 23.17 & 20.84 & 31.79 & 16.79 \\
$5.5-7.3$ & 8 & 22.52 & 30.88 & 20.27 & 26.10 & 31.61 & 23.43 & 25.13 & 19.24 \\
$7.6-10.4$ & 4 & 34.13 & 29.71 & 15.34 & 25.74 & 36.17 & 22.67 & 14.00 & 18.96 \\
\hline Promedio & 36 & 23.90 & 28.04 & 14.01 & 24.04 & 29.29 & 21.49 & 32.56 & 17.58 \\
\hline
\end{tabular}

atribuidos a una alta proporción de fibras meduladas (Villarroel, 1963), lo cual estaría afectando el valor promedio de FH.

Los mayores valores de DMF, CV y FH reportados en este estudio pueden atribuirse principalmente a efectos del sexo, localidad y manejo. En este estudio solo se evaluaron machos criados bajo un sistema mejorado de alimentación y manejo.

El DMF tiende a incrementarse con el incremento de la edad del animal, siendo más notable esta tendencia en los primeros años (McGregor y Butler, 2004; Gutiérrez et al., 2011). Similar patrón fue observado en el presente estudio para el DMF y FH, donde las correlaciones con la edad fueron positivas $(\mathrm{p}<0.01)$.

\section{Variabilidad Fenotípica de Fibras Medu- ladas}

El promedio del porcentaje de medulación total (PM) y su desviación estándar fue $67.44 \pm 18.30 \%$, valor cercano al $69.3 \pm 8.7 \%$ reportado por Hack et al. (1999) en alpacas de Australia, y similar al $68 \%$ reportado por Córdova (2015) en alpacas macho del Ecuador y al $68.5 \%$ por Contreras (2009) en alpacas macho de color blanco de Huancavelica. Por otra parte, el error están- dar del PM varió entre 1.13 y $2.04 \%$, siendo el promedio de $1.74 \%$, menor al $4.96 \%$ reportado por Contreras (2009).

Se observó una amplia variación fenotípica del PM entre animales, con valores extremos de 12.33 y $91.67 \%$, rango similar al de $5-90 \%$ reportado por McGregor (2006). Tanto el DMF como el PM presentan variaciones según las regiones corporales (Newman y Paterson, 1996; Córdova, 2015); sin embargo, en este estudio solo se evaluó el costillar medio, teniendo en consideración que es la región del vellón más representativa para el DMF. Para fines de selección por PM y otras características de las fibras meduladas, se sugiere que la región del flanco (ijar) es la región más representativa (Aylan-Parker y McGregor, 2002).

En la variación fenotípica del porcentaje de fibras meduladas no se consideró a la fibra fuertemente medulada. El porcentaje de fibra medulada fragmentada, discontinua y continua fue muy variable entre los animales del estudio (Cuadro 2, Figura 2). La variabilidad fenotípica también se observó mediante la superposición de las curvas del porcentaje de fibras no meduladas, meduladas fragmentadas, discontinuas y continuas en el rango de 10 y $45 \%$ (Figura 2). 


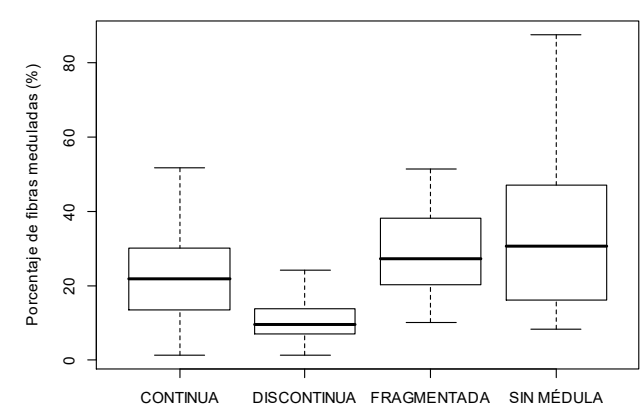

Figura 2. Variación del porcentaje (\%) de fibra medulada continua, discontinua, fragmentada y sin médula en vellón de alpacas Huacaya

Las fibras fuertemente meduladas se observaron en 15 de los 36 animales $(0.59 \pm$ $0.44 \%$ ), con valores cercanos al $0.41 \%$ reportado por Wurzinger et al. (2006) en el vellón de llamas machos de Bolivia, pero inferior al valor reportado por Villarroel (1963) en alpacas Huacaya.

El valor promedio y desviación estándar del porcentaje de fibra medulada continua fue $23.90 \pm 13.01 \%$, inferior al 28.46, $40.6 \mathrm{y}$ $32.50 \%$ reportados por Cruz et al. (2017), Villarroel (1963) y Trejo (1986), respectivamente, mientras que los valores extremos fueron de 1.17 y $65.50 \%$, similares a los valores reportados por Cruz et al. (2017). El valor para la fibra medulada discontinua fue de $14.01 \pm 10.14 \%$, valor cercano al $12.20 \mathrm{y}$ $14.9 \%$ reportados por Trejo (1986) y Villarroel (1963), respectivamente. El de la fibra medulada fragmentada fue de $29.29 \pm$ $11.67 \%$, superior al 13.9 y $17.17 \%$ reportados por Villarroel (1963) y Contreras (2009), respectivamente. Así mismo, para el caso de la fibra no medulada fue de $32.56 \pm 18.30 \%$, valor inferior al $38.93 \%$ reportado por Contreras (2009); sin embargo, Villarroel (1963) y Newman y Paterson (1996) reportaron valores de 21.1 y $18.3 \%$, respectivamente.

La reducción del porcentaje de fibras meduladas continuas y fuertemente meduladas, así como el incremento del porcentaje de fibras meduladas fragmentadas y no meduladas en el vellón de alpacas puede atribuirse a la selección dirigida para reducir el DMF que se practica en Pacomarca (Cervantes et al., 2010).

Por otra parte, se observó un incremento del PM con el incremento de la edad del animal $(p<0.01)$. La incidencia del PM fue más notable en animales viejos (entre 5 y 10 años) comparado a los animales jóvenes (entre 1 y 4 años). Similar tendencia fue reportada por McGregor (2006). La incidencia del porcentaje de fibras meduladas quizás sea afectada, además, por la frecuencia de esquila y la edad (Martinez et al., 1997; Lupton et al., 2006). Así mismo, se ha reportado que el número de folículos activos disminuye y algunos folículos secundarios comienzan a producir fibras meduladas con el incremento de la edad (Antonini et al., 2004; Khan et al., 2012).

Los mecanismos que dan origen a los tipos de médula no han sido dilucidados; sin embargo, se conoce que factores ambientales como la estación del año y la nutrición afectan la incidencia de fibras meduladas en cabras Angora (McGregor, 2018). Por otro lado, se ha reportado variabilidad genética aditiva para los caracteres relacionados a la medulación de la fibra, estimándose valores de heredabilidad que varían de moderada a alta (Sánchez et al., 2016; Cruz et al., 2017; Pinares et al., 2018). Además, se ha sugerido que la expresión de las fibras meduladas estaría controlada por muchos genes (Yu et al., 2011; Phua et al., 2015). La variabilidad fenotípica es importante para iniciar un programa de selección, pero la variabilidad genética aditiva es determinante en la respuesta a la selección por PM (Sánchez et al., 2016).

El diámetro en más del 50\% de fibras no meduladas fue menor a $18 \mu \mathrm{m}$, donde el $84 \%$ de estas fibras mostraron diámetros menores a $21 \mu \mathrm{m}$ y el $97 \%$ mostraron diámetros menores a $25 \mu \mathrm{m}$ (Cuadro 3, Figura 3). Por tanto, el rango de finura de fibras no meduladas sería entre 15 y $25 \mu \mathrm{m}$, similar al 
Cuadro 3. Proporción de fibra medulada continua, discontinua, fragmentada y no medulada, distribuida por intervalos de $3 \mu \mathrm{m}$ de diámetro en vellones de alpacas Huacaya

\begin{tabular}{ccccc}
\hline Diámetro $(\mu \mathrm{m})$ & $\begin{array}{c}\text { Continua } \\
\%\end{array}$ & $\begin{array}{c}\text { Discontinua } \\
\%\end{array}$ & $\begin{array}{c}\text { Fragmentada } \\
\%\end{array}$ & $\begin{array}{c}\text { No medulada } \\
\%\end{array}$ \\
\hline$<18$ & 1.34 & 2.71 & 13.77 & 51.03 \\
$18-21$ & 8.59 & 18.45 & 34.67 & 33.12 \\
$22-25$ & 23.42 & 34.97 & 33.31 & 12.79 \\
$26-29$ & 28.57 & 29.27 & 14.46 & 2.73 \\
$30-33$ & 20.65 & 11.68 & 3.03 & 0.25 \\
$34-37$ & 10.35 & 2.56 & 0.58 & 0.09 \\
$>38$ & 7.08 & 0.36 & 0.17 & 0.00 \\
\hline
\end{tabular}

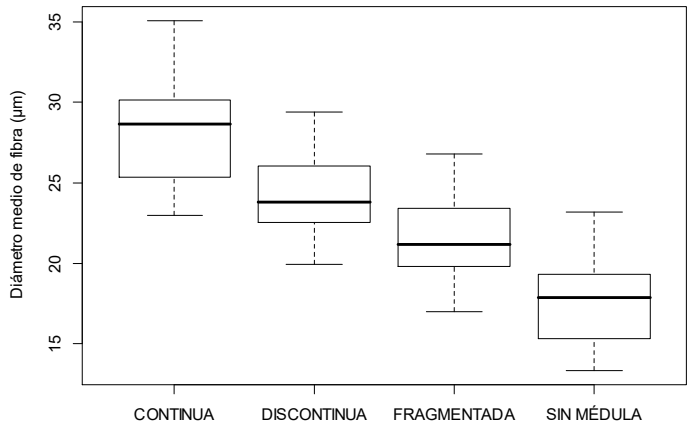

Figura 3. Variación del diámetro medio de fibra $(\mu \mathrm{m})$ medulada continua, discontinua, fragmentada y sin médula en vellón de alpacas Huacaya

rango reportado por Villarroel (1963) entre 15 y $20 \mu \mathrm{m}$. E1 96\% de las fibras meduladas fragmentadas mostraron diámetros menores a $30 \mu \mathrm{m}$ y el rango de finura para este grupo de fibras sería entre 18 y $30 \mu \mathrm{m}$, similar al rango reportado por Villarroel (1963) entre 20 y $30 \mu \mathrm{m}$. Los rangos de finura para las fibras meduladas discontinuas serían entre 18 y $33 \mu \mathrm{m}$ y para el grupo de fibras meduladas continuas sería entre 20 y $36 \mu \mathrm{m}$, diferente a los rangos reportado por Villarroel (1963) con valores mayores a 30 y $40 \mu \mathrm{m}$ para fibras meduladas discontinuas y continuas, respectivamente. Los resultados del presente estudio indican que en el rango entre 18 y $36 \mu \mathrm{m}$ se pudo observar fibras meduladas fragmentadas, discontinuas y continuas.
Por otro lado, se estimó la siguiente ecuación para la predicción el porcentaje de medulación total: $\mathrm{PM}=-24.63+4.17(\mathrm{DMF})$, con un coeficiente de determinación $\left(\mathrm{R}^{2}\right)$ de 0.64 , mayor al de 0.60 estimado por McGregor (2006). El coeficiente de regresión estimado fue superior al 3.23 estimado por McGregor (2006), diferencias que podrían atribuirse a las diferencias en tamaño y tipo de muestras.

\section{Correlaciones Fenotípicas}

Las correlaciones fenotípicas entre los caracteres fueron positivas y de magnitud intermedia a alta (Cuadro 4). La correlación fenotípica entre el DMF y el PM fue alta $(r=0.80)$, ligeramente superior al 0.77 reportado por McGregor (2006) y superior al 0.64 y 0.62 reportado por Córdova (2015) y Cordero et al. (2011), respectivamente. La correlación genética reportado por Pinares et al. (2018) fue positiva y alta (0.93) entre el diámetro individual de la fibra y la presencia de medulación, lo que sugiere una reducción del diámetro de fibra al seleccionar por el porcentaje de fibras meduladas. Sin embargo, para el diseño de estrategias de mejoramiento genético de la calidad de fibra de alpaca, se requiere calcular la correlación genética entre el DMF y el PM.

La correlación fenotípica entre el DMF y el porcentaje de fibras $<30 \mu \mathrm{m}$ fue -0.90 , similar al valor de -0.90 reportado por Arango (2016) 
Cuadro 4. Coeficientes de correlación fenotípica (r) entre los diámetros medios de fibra según tipo de medulación en el vellón de alpaca Huacaya

\begin{tabular}{lccc}
\hline Caracteres & \multicolumn{3}{c}{ DMF por tipo de médula } \\
\cline { 2 - 4 } & continua & discontinua & fragmentada \\
\hline DMF & 0.76 & 0.84 & 0.83 \\
DMF no medulada & 0.76 & 0.82 & 0.82 \\
DMF fragmentada & 0.92 & 0.93 & 1 \\
\hline
\end{tabular}

DMF: Diámetro medio de fibra

y diferente al de -0.99 y -0.75 reportado por Machaca et al. (2017) y Vásquez et al. (2015), respectivamente. La correlación entre el DMF y la FH fue 0.98 , similar al valor de 0.99 reportado por Vásquez et al. (2015).

Los valores de correlación fenotípica fueron de magnitud intermedia para el PM y el porcentaje de medulación continua $(0.74)$ para el porcentaje de fibras $<30 \mu \mathrm{m}$ y el PM (-0.55), y para el porcentaje de fibras $<30 \mu \mathrm{m}$ y el porcentaje de medulación continua $(-0.40)$. Estos valores permiten sugerir que al disminuir el PM por selección podrían disminuir los valores promedios del porcentaje de medulación continua y la proporción de fibras $<30 \mu \mathrm{m}$ se incrementaría en el vellón.

La correlación fenotípica entre el diámetro medio de fibra no medulada y medulada continua fue 0.76 , y entre el diámetro medio de fibra medulada fragmentada y medulada continua fue 0.92 , superior a los valores de 0.60 y 0.74 reportados por Martínez et al. (1997), respectivamente. Las correlaciones fenotípicas para el DMF del grupo de fibras meduladas fragmentadas, discontinuas y continuas fueron positivas y se relacionaron con la superposición de las curvas de distribución de los diámetros de las fibras meduladas fragmentadas, discontinuas y continuas.

\section{Conclusiones}

- Los vellones de la alpaca Huacaya contienen un alto porcentaje de fibras meduladas, con alta variabilidad fenotí- pica entre los animales y por el tipo de médula.

- Se encontró una correlación fenotípica favorable y positiva entre el diámetro medio de fibra y el porcentaje de medulación.

- Las fibras finas tienen una baja frecuencia de médulas fragmentadas y discontinuas, mientras que las fibras más gruesas tienen una mayor frecuencia de médulas discontinuas y continuas.

- La selección a favor de la reducción del porcentaje de fibras meduladas continuas en el vellón podría contribuir a mejorar el confort y el valor textil de la fibra en las prendas de alpaca.

\section{Literatura Citada}

\section{Antonini M, Gonzales M, Valbonesi A.} 2004. Relationship between age and postnatal skin follicular development in three types of South American domestic camelids. Livest Prod Sci 90: 241-246. doi: 10.1016/j.livprodsci.2004.06.001

2. Arango SJS. 2016. Variación del factor confort en vellones de alpaca Huacaya con relación al sexo y edad. Tesis de Ingeniero Zootecnista. Lima: Univ. Nacional Agraria La Molina. 47 p.

3. Aylan-Parker J, McGregor BA. 2002. Optimising sampling techniques and estimating sampling variance of fleece quality attributes in alpacas. Small Ruminant Res 44: 53-64. doi: 10.1016/ S0921-4488(02)00038-X 
4. Butler KL, Dolling M. 1995. Spinning fineness of wool. J Text I 85: 164-166. doi: 10.1080/00405009508631319

5. Cervantes I, Pérez-Cabal MA, Morante R, Burgos A, Salgado C, Nieto B, Goyache F, et al. 2010. Genetic parameters and relationships between fibre and type traits in two breeds of Peruvian alpacas. Small Ruminant Res 88: 6-11. doi: 10.1016/j.smallrumres.2009.10.016

6. Contreras A. 2009. Estructura cuticular y características físicas de la fibra de alpaca Huacaya (Vicugna pacos) de color blanco en la región de Huancavelica. Tesis de Ingeniero Zootecnista. Huancavelica: Univ. Nacional de Huancavelica. 90 p.

7. Cordero A, Contreras J, Mayhua P, Jurado M, Castrejón M. 2011. Correlaciones fenotípicas entre características productivas en alpacas Huacaya. Rev Inv Vet Perú 22: 15-21. doi: 10.15381/ rivep.v22i1.114

8. Córdova ML. 2015. Comparación de la calidad de las fibras de Vicugna pacos (alpaca) y Lama glama (llama). Tesis de Ingeniero Zootecnista. Riobamba: Escuela Superior Politécnica de Chimborazo. $113 \mathrm{p}$.

9. Cruz A, Morante $R$, Cervantes I, Burgos A, Gutiérrez JP. 2017. Genetic parameters of medullation percentage in alpacas. In: $68^{\text {th }}$ Annual Meeting of the European Federation of Animal Science. Tallinn, Estonia.

10. González H, León C, Rosadoo R, García W, Gavidia C. 2008. Evaluación de un método numérico de medición del diámetro de la fibra de alpaca. Rev Inv Vet Perú 19: 1-8. doi: 10.15381/ rivep.v19i1.641

11. Gutiérrez JP, Varona L, Pun A, Morante R, Burgos A, Cervantes I, Pérez-Cabal MA. 2011. Genetic parameters for growth of fiber diameter in alpacas. J Anim Sci 89: 2310-2315. doi: 10.2527/jas.2010-3746

12. Hack W, McGregor B, Ponzoni R, Judson G, Carmicheal I, Hubbard D. 1999. Australian alpaca fibre: improving productivity and marketing. Rural Industries Research and Development Corporation Research RIRDC N ${ }^{\circ}$ 99/140.

13. IWTO-8. 2011. Fiber diameter distribution parameters and percentage of medullated fibers in wool and other animal fibers by the projection micros-cope. In: Wto Red Book Specifications. International Wool Textile Organization.

14. Khan MJ, Abbas A, Ayaz M, Naeem M, Akhater MS, Soomro MH. 2012. Factors affecting wool quality and quantity in sheep. Afr J Biotechnol 11: 13761-13766.

15. Laime F, Pinares R, Paucara V, Machaca V, Quispe EC. 2016. Características tecnológicas de la fibra de llama (Lama glama) Chaku antes y después de descerdar. Rev Inv Vet Perú 27: 209217. doi: 10.15381/rivep.v27i2.11643

16. Lupton C, Mccoll A, Stobart R. 2006. Fiber characteristics of the Huacaya alpaca. Small Ruminant Res 64: 211-224. doi: 10.1016/j.smallrumres.2005.04.023

17. Machaca V, Bustinza AV, Corredor FA, Paucara V, Quispe EC, Machaca R. 2017. Características de la fibra de alpaca Huacaya de Cotaruse, Apurímac, Perú. Rev Inv Vet Perú 28: 843-851. doi: 10.15381/rivep.v28i4.13889

18. Martínez $Z$, Iñiguez LC, Rodríguez T. 1997. Influence of effects on quality traits and relationships between traits of the llama fleece. Small Ruminant Res 24: 203-212. doi: 10.1016/S0921-4488(96)00925-X

19. McGregor B. 2006. Production attributes and relative value of alpaca fleeces in southern Australia and implications for industry development. Small Ruminant Res 61: 93-111. doi: 10.1016/j.smallrumres.2005.07.001

20. McGregor BA, Butler. 2004. Sources of variation in fibre diameter attributes of Australian alpacas and implications for fleece evaluation and animal selection. Aust J Agr Res 55: 433-442. doi: 10.1071/AR03073

21. McGregor BA, Butler KL, Ferguson MB. 2013. The relationship of the incidence of medullated fibres to the 
dimensional properties of Mohair over the lifetime of Angora goats. Small Ruminant Res 115: 40-50. doi: 10.1016/j.smallrumres.2013.08.010

22. Moore KE, Blache D, Maloney SK. 2011. Fibre diameter and insulation in alpacas: the biophysical implications. Small Ruminant Res 96: 165-172. doi: 10.1016/j.smallrumres.2011.01.012

23. Newman SAN, Paterson DJ. 1996. Variation in fleece characteristics over the body of alpaca. Proc New Zeal Soc An 56: 338-341.

24. Pinares R, Gutiérrez GA, Cruz A, Morante R, Cervantes I, Burgos A, Gutiérrez JP. 2018. Heritability of individual fiber medullation in Peruvian alpacas. Small Ruminant Res 165: 93100. doi: $10.1016 /$ j.smallrumres.2018.04.007

25. Phua SH, Cullen NG, Dodds KG, Scobie DR, Bray AR. 2015. An ovine quantitative trait locus affecting fibre opacity in wool. Small Ruminant Res 130: 122-126.

26. Quispe EC. 2010. Estimación del progreso genético de seis esquemas de selección en alpacas (Vicugna pacos L) Huacaya con tres modelos de evaluación en la región altoandina de Huancavelica. Tesis Doctoral. Lima: Univ. Nacional Agraria La Molina. $144 \mathrm{p}$.

27. $R$ Core Team. 2017. R: a language and environment for statistical computing. $R$ Foundation for Statistical Computing, Vienna, Austria. [Internet]. Disponible en: http://www.R-project.org/

28. Sánchez AL, Urioste JI, Peñagaricano $F$, Neimaur K, Sienra I, Naya $H$, Kremer R. 2016. Genetic parameters of objectionable fibers and of their associations with fleece traits in Corriedale sheep. J Anim Sci 94: 13-20. doi: 10.2527/ jas.2015-9619

29. Scobie DR, Grosvenor AJ, Bray AR, Tandon SK, Meade WJ, Cooper AMB. 2015. A review of wool fibre variation across the body of sheep and the effects on wool processing. Small Ruminant Res 133: 53-53. doi: 10.1016/j.smallrumres.2015.10.025

30. Sienra I, Neimaur K, Kremer $R$, Urioste JI. 2011. Medullated fibers and fleece characteristics in Corriedale hoggets from two flocks in Uruguay. Anim Prod Sci 51: 1034-1038. doi: 10.1071/AN11031

31. Trejo W. 1986. Estudio de la correlación fenotípica entre el diámetro de fibra y la escala de colores en Alpacas Huacaya. Tesis de Ingeniero Zootec-nista. Lima: Univ. Nacional Agraria La Molina. 67 p.

32. Vásquez RA, Gómez OE, Quispe EC. 2015. Características tecnológicas de la fibra blanca de alpaca Huacaya en la zona altoandina de Apurímac. Rev Inv Vet Perú 26: 213-222. doi: 10.15381/ rivep.v26i2.11020

33. Villarroel J. 1963. Un estudio de la fibra de alpaca. Anales Científicos UNALM 1: 246-274.

34. Wang L, Singh A, Wang X. 2008. Dehairing Australian alpaca fibres with a cashmere dehairing machine. J Text I 99: 539-544. doi: 10.1080/00405000701609308

35. Wang HM, Xin L, Wang X. 2005. Internal structure and pigment granules in coloured alpaca fibers. Fiber Polym 6: 263-268. doi: 10.1007/BF02875652

36. Wurzinger M, Delgado J, Nürnberg M, Valle Zárate A, Stemmer A, Ugarte G, Sölkner J. 2006. Genetic and nongenetic factors influencing fibre quality of Bolivian llamas. Small Ruminant Res 61: 131-139. doi: 10.1016/j.smallrumres.2005.07.004

37. Yu Z, Wildermoth JE, Wallace OAM, Gordon SW, Maqbool NJ, Maclean PH, Nixon AJ, et al. 2011. Annotation of sheep keratin intermediate filament genes and theirpatterns of expression. Exp Dermatol 20: 582-588. doi: 10.1111/ j.1600-0625.2011.01274.x 